PROCEEDINGS OF THE

AMERICAN MATHEMATICAL SOCIETY

Volume 136, Number 9, September 2008, Pages 3331-3338

S 0002-9939(08)09237-X

Article electronically published on May 5, 2008

\title{
ENTROPY-COST INEQUALITIES FOR DIFFUSION SEMIGROUPS WITH CURVATURE UNBOUNDED BELOW
}

\author{
FENG-YU WANG \\ (Communicated by Richard C. Bradley)
}

\begin{abstract}
The weighted log-Sobolev inequality and the entropy-cost inequality are established for a class of diffusion semigroups with curvature unbounded below. Concrete examples are presented to illustrate the main results.
\end{abstract}

\section{INTRODUCTION}

Let $(M, g)$ be a $d$-dimensional connected complete Riemannian manifold either with convex boundary or without boundary, and let $L:=\Delta+\nabla V$ for some second differentiable function $V$ on $M$. Let $K \in C(M)$ be such that

$$
\left(\operatorname{Ric}-\operatorname{Hess}_{V}\right)(X, X) \geq-K(x), \quad X \in T_{x} M, x \in M .
$$

When $K$ is constant (i.e. the curvature of $L$ is bounded below), many properties of the corresponding diffusion semigroup $P_{t}$ have been well described in the literature; see e.g. 2, 14 and references therein for gradient estimates and semigroup functional inequalities, 12, 13, 11, for a dimension-free Harnack inequality and its applications to various contractivity properties, [10] for estimates of probability distances, and 4 for an HWI (entropy-cost-information) inequality.

Recently, the dimension-free Harnack inequality was extended in 1 to the case that $K \leq c\left(1+\rho_{o}^{2}\right)$ for some constant $c>0$ and $o \in M$, where $\rho_{o}$ is the Riemannian distance function to $o$. The main purpose of this paper is to study the log-Sobolev inequality and the entropy-cost inequality for unbounded $K$. To this end, we assume that $\mu(\mathrm{d} x):=\mathrm{e}^{V(x)} \mathrm{d} x$ is a probability measure so that the $L$-diffusion process is nonexplosive. Let $\rho$ be a distance on $M$. For any probability measure $\nu$ on $M$, define the $L^{2}$-Wasserstein distance (or the $L^{2}$-transportation cost) between $\mu$ and $\nu$ w.r.t. $\rho$ :

$$
W_{2}^{\rho}(\mu, \nu):=\inf _{J \in \mathcal{C}(\mu, \nu)}\left\{\int_{M \times M} \rho(x, y)^{2} J(\mathrm{~d} x, \mathrm{~d} y)\right\}^{1 / 2},
$$

where $\mathcal{C}(\mu, \nu)$ is the set of all couplings for $\mu$ and $\nu$.

Received by the editors August 15, 2006, and, in revised form, April 12, 2007.

2000 Mathematics Subject Classification. Primary 58G32, 60J60.

Key words and phrases. Curvature, diffusion semigroup, entropy-cost inequality, log-Sobolev inequality.

This work was supported in part by the Creative Research Group Fund of the National Natural Science Foundation of China (No. 10121101) and RFDP(20040027009).

(C)2008 American Mathematical Society 
To derive the entropy-cost inequality, we shall make use of the following generalized gradient estimate:

$$
\tilde{g}\left(\tilde{\nabla} P_{t} f, \tilde{\nabla} P_{t} f\right) \leq \alpha(t) P_{t}|\nabla f|^{2}, \quad t \geq 0, f \in C_{b}^{1}(M),
$$

where $\tilde{g}$ is another metric on $M$ and $\tilde{\nabla}$ is the corresponding gradient operator. When $\tilde{g}$ is much smaller, this condition is much weaker than known ones for $\tilde{g}=g$ in the literature (cf. [2]).

Theorem 1.1. If there exist measurable $\alpha:[0, \infty) \rightarrow(0, \infty)$ and a metric $\tilde{g}$ such that (1.2) holds, then

$$
\mu\left(\left(P_{t} f\right) \log P_{t} f\right) \leq \frac{W_{2}^{\tilde{\rho}}(f \mu, \mu)^{2}}{4 \int_{0}^{t} \alpha(s)^{-1} \mathrm{~d} s}, \quad t>0, f \geq 0, \mu(f)=1,
$$

where $\tilde{\rho}$ is the Riemannian distance induced by the metric $\tilde{g}$.

Next, to derive the log-Sobolev type inequality, we need the following condition on curvature:

$$
x \mapsto P_{t}^{K} 1(x):=\mathbb{E}^{x} \exp \left[2 \int_{0}^{t} K\left(x_{s}\right) \mathrm{d} s\right] \text { is locally bounded, }
$$

where $x_{s}$ is the $L$-diffusion process. This condition is satisfied by many unbounded above functions $K$ (cf. examples below).

Theorem 1.2. If (1.4) holds, then

$$
\mu\left(f^{2} \log f^{2}\right) \leq \mu\left(\left(P_{t} f^{2}\right) \log P_{t} f^{2}\right)+4 \int_{0}^{t} \mu\left(\left(P_{s}^{K} 1\right)|\nabla f|^{2}\right) \mathrm{d} s, \quad f \in C_{0}^{1}(M), t \geq 0 .
$$

If, in particular,

$$
A(x):=\int_{0}^{\infty} P_{t}^{K} 1(x) \mathrm{d} t<\infty, \quad x \in M
$$

is locally bounded, then the following weighted log-Sobolev inequality holds:

$$
\mu\left(f^{2} \log f^{2}\right) \leq 4 \mu\left(A|\nabla f|^{2}\right), \quad f \in C_{0}^{1}(M), \mu\left(f^{2}\right)=1 .
$$

Remark. (a) If $K$ is constant, then (1.4) holds for $\tilde{g}=g$ and $\alpha(t)=\mathrm{e}^{2 K t}$ (cf. 2]). Thus, (1.3) reduces to

$$
\mu\left(\left(P_{t} f\right) \log P_{t} f\right) \leq \frac{2 K W_{2}^{\rho}(f \mu, \mu)^{2}}{1-\exp [-2 K t]}, \quad t>0, f \geq 0, \mu(f)=1,
$$

where $\rho$ is the Riemannian distance induced by $g$. This coincides with an inequality in [4, which, together with (1.5) for $P_{s}^{K} 1=\mathrm{e}^{2 s K}$, implies the so-called HWI inequality therein.

(b) If, in particular, $K<0$, then (1.6) reduces to

$$
\mu\left(f^{2} \log f^{2}\right) \leq-\frac{2}{K} \mu\left(|\nabla f|^{2}\right), \quad \mu\left(f^{2}\right)=1,
$$

which follows from the well known Bakry-Emery criterion ([3]).

To see that Theorems 1.1 and 1.2 apply to many unbounded below curvature situations, we introduce the following consequences with more explicit conditions on curvature. Let $\rho_{o}$ be the Riemannian distance to a fixed point $o \in M$. By the 
Laplacian comparison theorem, there exists a nonnegative function $\gamma \in C([0, \infty))$ such that

$$
L \rho_{o}(x) \leq \gamma \circ \rho_{o}(x), \quad \rho_{o}(x) \geq 1, x \notin \operatorname{cut}(o),
$$

where $\operatorname{cut}(o)$ is the cut-locus of $o$ (see (1.12) below for a concrete choice of $\gamma$ ). We note that since $L \rho_{o} \rightarrow \infty$ as $\rho_{o} \rightarrow 0$, we exclude in (1.7) a neighborhood of $o$.

Corollary 1.3. Let $\gamma \geq 0$ satisfy (1.7). If there exists $C>0$ such that

$$
2 \gamma(r)^{2}+\gamma^{\prime}(r) \leq C+C \int_{0}^{r} \gamma(s) \mathrm{d} s, \quad r \geq 0
$$

and

$$
\lim _{\rho_{o} \rightarrow \infty} \frac{K^{+}}{\int_{0}^{\rho_{o}} \gamma(s) \mathrm{d} s}=0
$$

then there exist constants $\delta, c>0$ such that

$$
P_{t}^{K} 1 \leq \exp \left[\int_{0}^{\rho_{o}} \gamma(s) \mathrm{d} s+c(\delta, t)+c t\right], \quad t \geq 0,
$$

where $c(\delta, t):=\sup _{M}\left\{2 K t-\mathrm{e}^{-\delta t} \int_{0}^{\rho_{o}} \gamma(s) \mathrm{d} s\right\}<\infty$ due to (1.9). Consequently, (1.4) is fulfilled and (1.2) is satisfied with

$$
\alpha(s):=\mathrm{e}^{c(\delta, s)+c s}
$$

and

$$
\tilde{g}:=\exp \left[\int_{0}^{\rho_{o}} \gamma(s) \mathrm{d} s\right] g,
$$

so that (1.3) holds for this $\alpha$ and the Riemannian distance induced by $\tilde{g}$.

Next, we present a result on the weighted log-Sobolev inequality (1.6) for operators with curvature unbounded below.

Corollary 1.4. Let $W \in C^{2}(M)$ be nonnegative with $\|\nabla V\|_{\infty}<\infty$, and $\gamma \in C(M)$ with $\gamma \rightarrow \infty$ as $\rho_{o} \rightarrow \infty$, such that

$$
L W \leq \theta-\gamma, \quad K \leq c_{0}+c_{1} \gamma
$$

for some constants $\theta, c_{0}, c_{1}>0$. Then for any $\varepsilon>0$ there exists $c(\varepsilon)>0$ such that (1.6) holds for

$$
A(x):=c(\varepsilon) \mathrm{e}^{2\left(c_{1}+\varepsilon\right) W(x)}, \quad x \in M .
$$

To illustrate our results, we present some examples below. To this end, we first observe that

$$
L \rho_{o}(x) \leq C+\int_{0}^{\rho_{o}(x)} K\left(\eta_{s}\right) \mathrm{d} s, \quad \rho_{o}(x) \geq 1, x \notin \operatorname{cut}(o)
$$

holds for $C:=d-1+\sup _{\rho_{o} \leq 1}|\nabla V|$ and $\eta:\left[0, \rho_{o}(x)\right] \rightarrow M$ the minimal geodesic from $o$ to $x$. Indeed, let $\left\{U_{i}: 1 \leq i \leq d-1\right\}$ be constant vector fields along $\eta$ such that $\left\{\dot{\eta}, U_{1}, \cdots, U_{d-1}\right\}$ is an orthonormal basis. For any differentiable function $h$ with $h(0)=0, h\left(\rho_{o}(x)\right)=1$, using $\left\{h(s) U_{i}\right\}$ to replace the Jacobi fields in the second variational formula for $\Delta \rho_{o}$, and applying the index lemma (see e.g. [5]), we obtain

$$
\Delta \rho_{o} \leq \int_{0}^{\rho_{o}(x)}\left\{(d-1) \dot{h}(s)^{2}-h(s)^{2} \operatorname{Ric}(\dot{\eta}(s), \dot{\eta}(s))\right\} \mathrm{d} s .
$$


Taking $h(s)=s \wedge 1$, we arrive at

$$
\begin{aligned}
L \rho_{o}(x) & \leq \int_{0}^{\rho_{o}(x)}\left\{\frac{\mathrm{d}}{\mathrm{d} s}\left\langle\nabla V\left(\eta_{s}\right), h(s) \dot{\eta}(s)\right\rangle+(d-1) \dot{h}(s)^{2}-h(s)^{2} \operatorname{Ric}(\dot{\eta}(s), \dot{\eta}(s))\right\} \mathrm{d} s \\
& \leq d-1+\sup _{\rho_{o} \leq 1}|\nabla V|-\int_{0}^{\rho_{o}(x)} h(s)^{2}\left\{\operatorname{Hess}_{V}(\dot{\eta}(s), \dot{\eta}(s))-\operatorname{Ric}(\dot{\eta}(s), \dot{\eta}(s))\right\} \mathrm{d} s .
\end{aligned}
$$

This implies (1.12) due to (1.1).

Example 1.1. Let $M$ be a Cartan-Hadamard manifold so that $\rho_{o}$ is smooth on $M \backslash\{o\}$. By (1.12), (1.11) holds for any $W \in C^{2}(M)$ with $W=\rho_{o}$ when $\rho_{o} \geq 1$, and $\gamma=-\int_{0}^{\rho_{o}} K\left(\eta_{s}\right) \mathrm{d} s$, provided

$$
\lim _{\rho_{o}(x) \rightarrow \infty} \int_{0}^{\rho_{o}(x)} K\left(\eta_{s}\right) \mathrm{d} s=-\infty, \quad \limsup _{\rho_{o}(x) \rightarrow \infty} \frac{K(x)}{-\int_{0}^{\rho_{o}(x)} K\left(\eta_{s}\right) \mathrm{d} s}<\infty .
$$

In particular, assuming Ric $\geq-R \circ \rho_{o}$ for some nonnegative increasing function $R$ with

$$
\limsup _{s \rightarrow \infty} R(s) / s<\infty,
$$

(1.14) holds for $V=-H \circ \rho_{o}$ when e.g. $H(0)=H^{\prime}(0)=0$ and

$$
H^{\prime \prime}(s):=2-\sum_{n=1}^{\infty} n 1_{\left[n, n+n^{-1}\right]}(s) \geq 1-x, \quad x \geq 0 .
$$

This follows since taking

$$
h(s)=\left(s-\rho_{o}+\left(R \circ \rho_{o}+1\right)^{-1 / 2}\right)^{+} \beta R \circ \rho_{o}+1
$$

in (1.13) we obtain

$$
\Delta \rho_{o} \leq c\left(1+\beta R \circ \rho_{o}\right), \quad \rho_{o} \geq 1,
$$

for some constant $c>0$, and by (1.15) and the Hessian comparison theorem (cf. [5]),

$$
\left\langle\nabla V, \nabla \rho_{o}\right\rangle=-H^{\prime} \circ \rho_{o} \leq-\rho_{o}, \quad \operatorname{Hess}_{V} \leq-H^{\prime \prime} \circ \rho_{o} \leq \rho_{o}-1 .
$$

Example 1.2. If there exists a constant $c_{0}>0$ such that

$$
\text { Ric } \geq-c_{0}\left(1+\rho_{o}^{2}\right), \quad\left\langle\nabla V, \nabla \rho_{o}\right\rangle \leq c_{0}\left(1+\rho_{o}\right),
$$

then (1.16) with $R(s)=c_{0}\left(1+s^{2}\right)$ implies (1.7) for $\gamma(s):=c_{1} s$ for some $c_{1}>0$, which satisfies (1.8). Therefore, assertions in Corollary 1.3 hold provided $K^{+} \rho_{o}^{-2} \rightarrow$ 0 as $\rho_{o} \rightarrow \infty$.

Example 1.3. Let $V \in C^{2}\left(\mathbb{R}^{d}\right)$ such that $\mu(\mathrm{d} x):=\mathrm{e}^{V(x)} \mathrm{d} x$ is a probability measure. Consider $L:=\sum_{i, j=1}^{d} a_{i j} \partial_{i} \partial_{j}+\sum_{i=1}^{d} b_{i} \partial_{i}$ on $\mathbb{R}^{d}$, where $\left(a_{i j}\right)$ is nondegenerated and $b_{i}=\sum_{j=1}^{d}\left(\partial_{j} a_{i j}+a_{i j} \partial_{j} V\right)$. Then $L$ is symmetric w.r.t. $\mu(\mathrm{d} x):=$ $\mathrm{e}^{V(x)} \mathrm{d} x$ (cf. [6]). Let $\sigma:=ß a$. If there exists $\lambda \in \mathbb{R}$ such that

$$
\|\sigma(x)-\sigma(y)\|^{2}+\langle x-y, b(x)-b(y)\rangle \leq \lambda|x-y|^{2}, \quad x, y \in \mathbb{R}^{d},
$$

where $\|\cdot\|$ is the Hilbert-Schmidt norm on $\mathbb{R}^{d}$, then according to the proof of Theorem 4.13 in [6]

$$
\left|\nabla P_{t} f\right|^{2} \leq \mathrm{e}^{2 \lambda t} P_{t}|\nabla f|^{2}, \quad t \geq 0 .
$$


Therefore, if $a \geq \lambda_{0} I$ for some constant $\lambda_{0}>0$, then

$$
\left|\nabla P_{t} f\right|^{2} \leq \frac{\mathrm{e}^{2 \lambda t}}{\lambda_{0}} P_{t}\langle a \nabla f, \nabla f\rangle
$$

By this and Theorem 1.1 we arrive at

$$
\mu\left(\left(P_{t} f\right) \log P_{t} f\right) \leq \frac{\lambda W_{2}(f \mu, \mu)^{2}}{2 \lambda_{0}\left(1-\mathrm{e}^{-2 \lambda t}\right)}, \quad t>0, f \geq 0, \mu(f)=1,
$$

where $W_{2}$ is induced by the usual Euclidean distance. If moreover $\lambda<0$, then $P_{t}$ converges exponentially to $\mu$ in entropy w.r.t. $W_{2}^{2}$. We remark that condition (1.17) is in general much simpler than the curvature condition induced by the metric $g:=a^{-1}$.

\section{Proofs}

Proof of Theorem 1.1. We follow the line of 4 but use the generalized gradient estimate (1.2) to replace the usual one. For any $t>0$ and $x, y \in M$, let $\eta:[0, t] \rightarrow$ $M$ be the minimal geodesic under $\tilde{g}$ from $x$ to $y$. We have $\tilde{g}\left(\dot{\eta}_{s}, \dot{\eta}_{s}\right)^{1 / 2}=\tilde{\rho}(x, y) / t$, where $\dot{\eta}$ is the tangent vector field along $\eta$. Let

$$
g(s):=\frac{t \int_{0}^{s} \alpha(r)^{-1} \mathrm{~d} r}{\int_{0}^{t} \alpha(r)^{-1} \mathrm{~d} r}, \quad s \in[0, t] .
$$

Then $g(0)=0$ and $g(t)=t$. It follows from (1.2) that

$$
\begin{aligned}
& \frac{\mathrm{d}}{\mathrm{d} s}\left(P_{s} \log P_{t-s} f\right)\left(\eta_{g(s)}\right)=-P_{s}\left|\nabla \log P_{t-s} f\right|^{2}\left(\eta_{g(s)}\right) \\
& +g^{\prime}(s) \tilde{g}\left(\tilde{\nabla} P_{s} \log P_{t-s} f\left(\eta_{g(s)}\right), \dot{\eta}_{g(s)}\right) \\
& \leq\left\{-P_{s}\left|\nabla \log P_{t-s} f\right|^{2}+g^{\prime}(s) t^{-1} \tilde{\rho}(x, y) \alpha(s)^{1 / 2}\left(P_{s}\left|\nabla \log P_{t-s} f\right|^{2}\right)^{1 / 2}\right\}\left(\eta_{g(s)}\right) \\
& \leq \frac{\alpha(s) g^{\prime}(s)^{2} \tilde{\rho}(x, y)^{2}}{4 t^{2}}=\frac{\tilde{\rho}(x, y)^{2}}{4 \alpha(s)\left(\int_{0}^{t} \alpha(r)^{-1} \mathrm{~d} r\right)^{2}} .
\end{aligned}
$$

Integrating w.r.t. $\mathrm{d} s$ over $[0, t]$, we arrive at

$$
P_{t} \log f(y) \leq \log P_{t} f(x)+\frac{\tilde{\rho}(x, y)^{2}}{4 \int_{0}^{t} \alpha(s)^{-1} \mathrm{~d} s} .
$$

Replacing $f$ by $P_{t} f$ and noting that

$$
\int_{M} \log P_{2 t} f \mathrm{~d} \mu \leq \log \mu(f)=0
$$

from the Monge-Kontorovich formula

$$
W_{2}^{\tilde{\rho}}(\mu, f \mu)^{2}=\sup \left\{\mu\left(f g_{2}\right)-\mu\left(g_{1}\right): g_{1}, g_{2} \in C_{b}(M), g_{2}(y) \leq g_{1}(x)+\tilde{\rho}(x, y)^{2}\right\},
$$

we obtain

$$
\mu\left(f P_{t} \log P_{t} f\right) \leq \frac{W_{2}^{\tilde{\rho}}(\mu, f \mu)^{2}}{4 \int_{0}^{t} \alpha(s)^{-1} \mathrm{~d} s} .
$$

This completes the proof by the symmetry of $P_{t}$ w.r.t. $\mu$. 
Proof of Theorem [1.2. By (1.4), the Feynman-Kac semigroup

$$
P_{t}^{K} f(x):=\mathbb{E}^{x} f\left(x_{t}\right) \mathrm{e}^{2 \int_{0}^{t} K\left(x_{s}\right) \mathrm{d} s}, \quad t \geq 0, x \in M,
$$

is well-defined in $L^{1}(\mu)$ for all $f \in C_{0}(M)$. Indeed, letting

$$
P_{t}^{K_{n}} f(x):=\mathbb{E}^{x} f\left(x_{t}\right) \mathrm{e}^{2 \int_{0}^{t} K\left(x_{s}\right) \wedge n \mathrm{~d} s}, \quad n \geq 1,
$$

we have

$$
\left|\mu\left(g P_{t}^{K} f\right)\right|=\lim _{n \rightarrow \infty}\left|\mu\left(g P_{t}^{K_{n}} f\right)\right|=\lim _{n \rightarrow \infty}\left|\mu\left(f P_{t}^{K_{n}} g\right)\right| \leq\|g\|_{\infty}\left(\sup _{\operatorname{supp} f} P_{t}^{K} 1\right) \mu(|f|)
$$

for all $f, g \in C_{0}(M)$. Thus, $P_{t}^{K} f \in L^{1}(\mu)$ and $\mu\left(\left|P_{t}^{K} f\right|\right) \leq \sup _{\operatorname{supp} f} P_{t}^{K} 1$. Next, the Bismut formula (cf. [8, Proposition A6(i)]) implies

$$
\left|\nabla P_{t} f(x)\right| \leq \mathbb{E}^{x}\left|\nabla f\left(x_{t}\right)\right| \mathrm{e}^{\int_{0}^{t} K\left(x_{s}\right) \mathrm{d} s} .
$$

Then for any $f \in C_{0}^{1}(M)$,

$$
\begin{aligned}
& \frac{\mathrm{d}}{\mathrm{d} s} P_{s}\left(P_{t-s} f^{2}\right) \log \left(P_{t-s} f^{2}\right)(x) \\
& \leq P_{s} \frac{\left|\nabla P_{t-s} f^{2}\right|^{2}}{P_{t-s} f^{2}}(x) \leq \mathbb{E}^{x} \frac{1}{P_{t-s} f^{2}\left(x_{s}\right)}\left(\mathbb{E}^{x_{s}}\left|\nabla f^{2}\right|\left(x_{t-s}\right) \mathrm{e}^{\int_{0}^{t-s} K\left(x_{r}\right) \mathrm{d} r}\right)^{2} \\
& \leq 4 \mathbb{E}^{x} \mathbb{E}^{x_{s}}|\nabla f|^{2}\left(x_{t-s}\right) \mathrm{e}^{2 \int_{0}^{t-s} K\left(x_{r}\right) \mathrm{d} r}=4 \mathbb{E}^{x} P_{t-s}^{K}|\nabla f|^{2}\left(x_{s}\right) .
\end{aligned}
$$

Thus,

$$
P_{t} f^{2} \log f^{2} \leq\left(P_{t} f^{2}\right) \log P_{t} f^{2}+4 \int_{0}^{t} P_{s} P_{t-s}^{K}|\nabla f|^{2} \mathrm{~d} s .
$$

Taking an integral w.r.t. $\mu$ and noting that $P_{t}^{K}$ is symmetric w.r.t. $\mu$, we prove (1.5). Finally, if $A$ is locally bounded, (1.6) follows from (1.5) and the dominated convergence theorem by noting that $P_{t} f^{2} \rightarrow \mu\left(f^{2}\right)=1$ in $L^{2}(\mu)$ as $t \rightarrow \infty$ according to the weak Poincaré inequality (cf. [1]).

Proof of Corollary 1.3. Let $h \in C^{\infty}[0, \infty)$ such that $\left.h\right|_{[0,1]}=0, h^{\prime} \geq 0$ and $h(r)=1$ for $r \geq 2$. By (1.7) and the Itô formula due to Kendall [9], we have

$(2.2) \mathrm{d} h\left(\rho_{o}\left(x_{t}\right)\right) \rho_{o}\left(x_{t}\right) \leq \beta 2\left\{h^{\prime}\left(\rho_{o}\left(x_{t}\right)\right) \rho_{o}\left(x_{t}\right)+h\left(\rho_{o}\left(x_{t}\right)\right)\right\} \mathrm{d} b_{t}+\left(c_{1}+\gamma\left(\rho_{o}\left(x_{t}\right)\right) \mathrm{d} t\right.$, where $b_{t}$ is the one-dimensional Brownian motion and $c_{1}>0$ is a constant. For any $\delta>0$, let

$$
F_{\delta}(t):=\exp \left[\mathrm{e}^{-\delta t} \int_{0}^{\rho_{o}\left(x_{t}\right)} h(s) \gamma(s) \mathrm{d} s\right], \quad t \geq 0 .
$$

It follows from (2.2) that

$\mathrm{d} F_{\delta}(t) \leq \mathrm{d} M_{t}+F_{\delta}(t) \mathrm{e}^{-\delta t}\left\{\gamma^{2}\left(\rho_{o}\left(x_{t}\right)\right)\left(1+\mathrm{e}^{-\delta t}\right)+\gamma^{\prime}\left(\rho_{o}\left(x_{t}\right)\right)-\delta \int_{0}^{\rho_{o}\left(x_{t}\right)} \gamma(s) \mathrm{d} s+c_{0}\right\} \mathrm{d} t$ for some constant $c_{0}>0$ and a local martingale $M_{t}$. Taking $\delta=C$ and combining this with (1.8), we obtain

$$
\mathrm{d} F_{\delta}(t) \leq \mathrm{d} M_{t}+\left(C+c_{0}\right) \mathrm{d} t .
$$

Thus, for $c:=C+c_{0}$,

$$
\mathbb{E}^{x} F_{\delta}(t) \leq F_{\delta}(0) \mathrm{e}^{c t}, \quad t \geq 0 .
$$

Thus, it follows from (1.9) that

$$
\mathbb{E}^{x} \mathrm{e}^{2 K\left(x_{s}\right) t} \leq \mathbb{E}^{x} F_{\delta}(s) \mathrm{e}^{c(\delta, t)} \leq F_{\delta}(0) \mathrm{e}^{c s+c(\delta, t)}, \quad t \geq s \geq 0 .
$$


Therefore,

$P_{t}^{K} 1(x):=\mathbb{E}^{x} \mathrm{e}^{t} 2 K\left(x_{s}\right) \mathrm{d} s \leq \frac{1}{t} \int_{0}^{t} \mathbb{E}^{x} \mathrm{e}^{2 K\left(x_{s}\right) t} \mathrm{~d} s \leq \exp \left[\int_{0}^{\rho_{o}(x)} \gamma(s) \mathrm{d} s+c t+c(\delta, t)\right]$.

This implies (1.10). Hence, (1.2) follows from (2.1) and the Schwartz inequality, and (1.3) follows from Theorem 1.1

Proof of Corollary 1.4, By (1.11),

$$
\mathrm{d} W\left(x_{t}\right) \leq \beta 2\left\langle\nabla W\left(x_{t}\right), u_{t}^{-1} \mathrm{~d} B_{t}\right\rangle+\left(\theta-\gamma \circ \rho_{o}\left(x_{t}\right)\right) \mathrm{d} t,
$$

where $B_{t}$ is the Brownian motion on $\mathbb{R}^{d}$ and $u_{t}$ is the horizontal $L$-diffusion process on the space of frame bundles. This implies

$$
\begin{aligned}
\mathbb{E}^{x} e^{\lambda \int_{0}^{t} \gamma \circ \rho_{o}\left(x_{s}\right) \mathrm{d} s} & \leq \mathbb{E}^{x} \mathrm{e}^{\lambda \theta t+\lambda W(x)+\beta 2 \int_{0}^{t}\left\langle\nabla W\left(x_{s}\right), u_{s}^{-1} \mathrm{~d} B_{s}\right\rangle} \\
& \leq \mathrm{e}^{\lambda \theta t+\lambda W(x)+\lambda^{2}\|\nabla W\|_{\infty}^{2} t}, \quad \lambda, t>0 .
\end{aligned}
$$

Thus, letting $c_{2}:=2\|\nabla W\|_{\infty}^{2}\left(c_{1}+\varepsilon\right)^{2}$, we obtain

$$
\mathbb{E}^{x} \mathrm{e}^{2\left(c_{1}+\varepsilon\right) \int_{0}^{t} \gamma \circ \rho_{o}\left(x_{s}\right) \mathrm{d} s} \leq \mathrm{e}^{2\left(c_{1}+\varepsilon\right) \theta t+2 c_{2} t+2\left(c_{1}+\varepsilon\right) W(x)}, \quad t \geq 0 .
$$

We will complete the proof in two steps.

(a) Assume, instead of the second inequality of (1.11), that

$$
K \leq\left(c_{1}+\varepsilon\right) \gamma \circ \rho_{o}-N
$$

for some constant $N>c_{2}+\theta\left(c_{1}+\varepsilon\right)$. Then, it follows from (2.3) that

$$
\begin{aligned}
& P_{t}^{K} 1(x):=\mathbb{E}^{x} \mathrm{e}^{\int_{0}^{t} 2 K\left(x_{s}\right) \mathrm{d} s} \leq \mathbb{E}^{x} \mathrm{e}^{2\left(c_{1}+\varepsilon\right) \int_{0}^{t} \gamma \circ \rho_{o}\left(x_{s}\right) \mathrm{d} s-2 N t} \\
& \leq \mathrm{e}^{2\left(c_{1}+\varepsilon\right) W(x)-2\left(N-\left(c_{1}+\varepsilon\right) \theta-c_{2}\right) t} .
\end{aligned}
$$

This implies the desired assertion.

(b) In general, for fixed $N>c_{2}+\theta\left(c_{1}+\varepsilon\right)$, we have

$$
u_{N}:=\inf \left\{s \geq 0: \int_{0}^{s}\left(N+c_{0}-\varepsilon \gamma(u)\right) \mathrm{d} u \geq 0\right\}<\infty
$$

since $\gamma(u) \rightarrow \infty$ as $u \rightarrow \infty$. Define

$$
V_{N}:=-\int_{0}^{\rho_{o}} \mathrm{~d} s \int_{0}^{s \wedge u_{N}}\left(N+c_{0}-\varepsilon \gamma(u)\right) \mathrm{d} u .
$$

Then $V_{N}$ is bounded and

$$
\left\langle\nabla V_{N}, \nabla \rho_{o}\right\rangle=-\int_{0}^{\rho_{o} \wedge u_{N}}\left(N+c_{0}-\varepsilon \gamma(u)\right) \mathrm{d} u \leq 0 .
$$

So, (1.11) holds for $L_{N}:=L+\nabla V_{N}$. Next, since $M$ is a Cartan-Hadamard, by the Hessian comparison theorem we have $\operatorname{Hess}_{V_{N}} \leq-\left(N+c_{0}-\varepsilon \gamma\left(\rho_{o}\right)\right) 1_{\left[0, u_{N}\right]}\left(\rho_{o}\right)$. Thus, by (1.11)

$$
\text { Ric }-\operatorname{Hess}_{V+V_{N}} \geq N+c_{0}-K-\varepsilon \gamma\left(\rho_{o}\right) \geq N-\left(c_{1}+\varepsilon\right) \gamma\left(\rho_{o}\right) .
$$

Therefore, (2.4) holds for $L_{N}$ in place of $L$. That is, by (a), (1.6) holds for $\mu_{N}:=$ $\mathrm{e}^{V_{N}} \mu / \mu\left(\mathrm{e}^{V_{N}}\right)$ in place of $\mu$. Then the proof is completed since the log-Sobolev inequality is stable under the bounded perturbation $V_{N}$. More precisely, if (1.6) holds for $\mu_{N}$ in place of $\mu$, it also holds for $\mu$ itself with constant $c(\varepsilon)$ in the definition of $A(x)$ replaced by $c(\varepsilon) \mathrm{e}^{2\left\|V_{N}\right\|_{\infty}}$; see e.g. [7, Remark 1.20]. 


\section{ACKNOWLEDGEMENT}

The author is grateful to the referee for a careful reading and valuable suggestions and corrections.

\section{REFERENCES}

[1] M. Arnaudon, A. Thalmaier and F.-Y. Wang, Harnack inequality and heat kernel estimate on manifolds with curvature unbounded below, Bull. Sci. Math. 130(2006), 223-233. MR2215664 (2007i:58032)

[2] D. Bakry, On Sobolev and logarithmic Sobolev inequalities for Markov semigroups, "New Trends in Stochastic Analysis" (Editors: K. D. Elworthy, S. Kusuoka, I. Shigekawa), Singapore: World Scientific, 1997. MR1654503 (99m:60110)

[3] D. Bakry and M. Emery, Hypercontractivité de semi-groupes de diffusion, C. R. Acad. Sci. Paris. Sér. I Math. 299(1984), 775-778. MR772092 (86f:60097)

[4] S. G. Bobkov, I. Gentil and M. Ledoux, Hypercontractivity of Hamilton-Jacobi equations, J. Math. Pures Appl. 80(2001), 669-696. MR.1846020 (2003b:47073)

[5] J. Cheeger and D. G. Ebin, Comparison Theorems in Riemannian Geometry, Amsterdam: North-Holland, 1975. MR0458335 (56:16538)

[6] M.-F. Chen and F.-Y. Wang, Estimation of spectral gap for elliptic operators, Trans. Amer. Math. Soc. 349(1997), 1239-1267. MR 1401516 (97h:35175)

[7] J. D. Deuschel and D. W. Stroock, Hypercontractivity and spectral gap of symmetric diffusion with applications to stochastic Ising models, J. Funct. Anal. 92(1990), 30-48. MR1064685 (91j:58174)

[8] K. D. Elworthy and X.-M. Li, Formulae for the derivatives of heat semigroups, J. Funct. Anal. 125(1994), 252-286. MR1297021 (95j:60087)

[9] W. S. Kendall, The radial part of Brownian motion on a manifold: a semimartingale property, Ann. Probab. 15(1987), no. 4, 1491-1500. MR905343 (88k:60151)

[10] M.-K. von Renesse and K.-T. Sturm, Transportation inequalities, gradient estimates, entropy and Ricci curvature, Comm. Pure Appl. Math. 58(2005), 923-940. MR2142879|(2006j:53048)

[11] M. Röckner and F.-Y. Wang, Supercontractivity and ultracontractivity for (non-symmetric) diffusion semigroups on manifolds, Forum Math. 15(2003), 893-921. MR2010284 (2005b:58059)

[12] F.-Y. Wang, Logarithmic Sobolev inequalities on noncompact Riemannian manifolds, Probability Theory Relat. Fields 109(1997), 417-424. MR1481127 (98i:58253)

[13] F.-Y. Wang, Harnack inequalities for log-Sobolev functions and estimates of log-Sobolev constants, Ann. Probab. 27(1999), 653-663. MR1698947 (2000i:58067)

[14] F.-Y. Wang, Equivalence of dimension-free Harnack inequality and curvature condition, Int. Equat. Operator Theory 48(2004), 547-552. MR:2047597 (2004m:58061)

School of Mathematical Sciences, Beijing Normal University, Beijing 100875, People's Republic of China - and - Department of Mathematics, Swansea University, SA2 8PP, Wales, United Kingdom

E-mail address: wangfy@bnu.edu.cn

E-mail address: F.Y.Wang@swansea.ac.uk 